\title{
QCD scaling violation at small $x$
}

\author{
R.K. Ellis ${ }^{a}$, F. Hautmann ${ }^{\text {b, }}$, B.R. Webber ${ }^{b, 1}$ \\ a Fermi National Accelerator Iaboratory, P.O. Box 500, Batavia, IL 60510, USA \\ ${ }^{b}$ Cavendish Laboratory, Department of Physics, University of Cambridge, Madingley Road, Cambridge CB3 OHE, UK \\ Received 17 January 1995 \\ Editor: P.V. Landshoff
}

\begin{abstract}
We investigate the evolution of parton densities at small values of the momentum fraction, $x$, by including resummed anomalous dimerisions in the renoumalization group equations. The resummation takes into account the leading-logarithmic contributions $\left(\alpha_{S} \ln x\right)^{k}$ given by the BFKL equation and the next-to-leading-logarithmic corrections from quark evolution: We present numerical results for the parton densities and the deep inelastic structure function $F_{2}$.
\end{abstract}

\section{Small-x processes in QCD perturbation theory}

Perturbative QCD provides a good description of hadronic collisions at large values of the centre-ofmass energy $\sqrt{S}$ and the transferred momentum $p_{r}$, in the region $\Lambda_{\mathrm{QCD}}^{2} \ll p_{t}^{2} \sim S[1]$. This description is based on the renormalization group analysis of physical cross sections in terms of universai parton densities and process-dependent coefficient functions. The parton densities $\hat{f}_{a}\left(x, \mu^{2}\right)\left(a=q_{i}, \bar{q}_{i}, g, i=\right.$ $1, \ldots, N_{f}, N_{f}$ being the number of active flavours) are functions of the parton momentum fraction $x$ and the hard scale $\mu^{2} \sim p_{t}^{2}$. In leading-twist order, they satisfy the evolution equations

$\frac{d f_{a}\left(\omega, \mu^{2}\right)}{d \ln \mu^{2}}=\sum_{b} \gamma_{a b}\left(\omega, \alpha_{S}\left(\mu^{2}\right)\right) f_{b}\left(\omega, \mu^{2}\right)$

\footnotetext{
I Research supported in part by the UK Particle Physics and Astronomy Research Council and the EC Programme "Human Capital and Mobility", Network "Physics at High Energy Colliders", contract CHRX-CT93-0357 (DG 12 COMA).
}

where $\omega$ is the moment variable conjugate to $x$,

$$
f_{a}\left(\omega, \mu^{2}\right)=\int_{0}^{1} d x x^{\omega} f_{a}\left(x, \mu^{2}\right),
$$

the evolution kernels $\gamma_{a b}\left(\omega, \alpha_{S}\right)$ are the appropriate anomalous dimensions, and the running coupling $\alpha_{S}$ is determined by

$$
\begin{aligned}
& \frac{d \alpha_{S}\left(\mu^{2}\right)}{d \ln \mu^{2}}=-b_{0} \alpha_{S}^{2}-b_{1} \alpha_{S}^{3}+\mathcal{O}\left(\alpha_{S}^{4}\right) \\
& \left(b_{0}=\frac{33-2 N_{f}}{12 \pi}, \quad b_{1}=\frac{153-19 N_{f}}{24 \pi^{2}}\right) .
\end{aligned}
$$

Scaling violation is systematically taken into account to leading, next-to-leading, etc, accuracy in $\ln \mu^{2}$ by expanding the anomalous dimensions to fixed order in $\alpha_{S}$,

$\gamma_{a b}\left(\omega, \alpha_{S}\right)=\gamma_{a b}^{(1)}(\omega) \alpha_{S}+\gamma_{a b}^{(2)}(\omega) \alpha_{S}^{2}+\mathcal{O}\left(\alpha_{S}^{3}\right)$

and using the corresponding expansions of the coefficient functions. 
At present and future high-energy colliders, a new perturbative regime opens up, characterized by sriall values of $x$ (when $\Lambda_{\mathrm{QCD}}^{2} \ll p_{t}^{2} \ll S$ ). In particular, plentiful data on deep inelastic lepton scattering at low $x$ are coming from the HERA ep collider and fixedtarget experiments [2]. In the small- $x$ regime, novel dynamical effects enter [3] as a consequence of the presence of the two very different large scales $p_{t}^{2}$ and $S$. The new effects are related to the emission of many gluons, widely separated in rapidity and disordered in transverse momentum. They lead to logarithmic enhancements $\left(\alpha_{S} \ln x\right)^{k}$. in higher-loop contributions to the perturbative expansions of physical cross sections. Correspondingly, the anomalous dimensions contain singularities at $\omega \rightarrow 0$ to all orders in perturbation theory, as follows

$$
\begin{aligned}
& \gamma_{a b}\left(\omega, \alpha_{S}\right)=\sum_{k=1}^{\infty}\left(\frac{\alpha_{S}}{\omega}\right)^{k} A_{a b}^{(k)}+\sum_{k=0}^{\infty} \alpha_{S}\left(\frac{\alpha_{S}}{\omega}\right)^{k} B_{a b}^{(k)} \\
& +\mathcal{O}\left(\alpha_{S}^{2}\left(\alpha_{S} / \omega\right)^{k}\right)
\end{aligned}
$$

(analogous singularities show up in the coefficient functions as well). These contributions may spoil the convergence of the perturbative expansion at small $x$. Indeed, numerical studies in fixed-order perturbation theory have already found this instability in the $x$ range accessible at HERA energies [4]. The singular terms should be summed to all orders in perturbation theory to obtain reliable predictions.

Eq. (5) may be taken to define the logarithmic hierarchy at small $x$. We shall refer to the first tower of terms $A^{(k)}$ as the leading-logarithmic $(L(x))$ series, the second tower $B^{(k)}$ as the next-to-leadinglogarithmic $(\mathrm{NL}(x))$ series, and so on. To $\mathrm{L}(x)$ accuracy, small- $x$ contributions are resummed by the BFKL equation [5]. Beyond the leading logaritims, the study of small- $x$ phenomena has been the object of much recent effort, including calculational programmes to compute $\mathcal{O}\left(\alpha_{S}\right)$-corrections to the kernel of the BFKL equation $[6,7]$, as well as phenomenological models incorporating certain classes of subleading effects into that equation [8-10]. At this level, there is an interplay between small- $x$ dynamics and the mass singularities associated with the low transverse momentum region. High-energy factorization [11] provides a consistent way of combining small- $x$ resummation and leading-twist factorization of mass singularities. Next-to-leading results have been obtained in Refs. [12,13] using this method.

The purpose of the present work is to investigate quantitatively the impact of small- $x$ dynamics on the evolution of parton densities, and, in particular, on scaling violation in deep inelastic scattering. To do this, we use resummed evolution kernels, and makc predictions for the parton densities by solving the resulting renormalization group equations. Note that higher-twist effects, such as unitarity corrections at asymptotic energies $[9,14]$, are not dealt with in this treatment. Also, as no attempt is made to extend the BFKL equation to sub-leading orders, our results are not absolute predictions, but depend upon non-perturbative input densities.

The system of evolution equations ( 1 ) can be separated into flavour singlet and non-singlet components. It can be shown that the latter are not subject to small- $x$ corrections of the type (5). In what follows it will always be understood that the non-singlet equations are treated in the standard way using the expansion (4) up to two-loop accuracy $[15,16]$. The enhanced small-x contributions are all associated with the singlet sector. The singlet evolution equations can be written in the matrix form

$\frac{d}{d \ln \mu^{2}}\left(\begin{array}{l}f_{S} \\ f_{g}\end{array}\right)=\left(\begin{array}{ll}\gamma_{S S} & \gamma_{S g} \\ \gamma_{g s} & \gamma_{g g}\end{array}\right)\left(\begin{array}{l}f_{S} \\ f_{g}\end{array}\right)$

where the quark singlet density is defined as $f_{S}=$ $\sum_{i=1}^{N}\left(f_{q_{i}}+f_{\bar{q}_{i}}\right)$. In the next section we summarize the main resummed results on the matrix kernel in Eq. (6). In Section 3 we match them with fixed-order contributions of the type (4), find the corresponding solutions of the evolution equations, and determine predictions for the deep inelastic structure function $F_{2}$.

In this Letter we concentrate on presenting the main results of our analysis. A more detailed discussion will be reported elsewhere [17].

\section{Anomalous dimensions at small $x$}

At leading logarithmic $(\mathrm{L}(x))$ level, $\mathrm{QCD}$ evolution is only fed by the gluon channel, and the resummation of the $\mathcal{O}\left(\alpha_{S} / \omega\right)^{k}$ contributions to the gluon anomalous dimensions is accomplished by the BFKL equation [5]. The resulting structure of the singlet anomalous dimension matrix is 

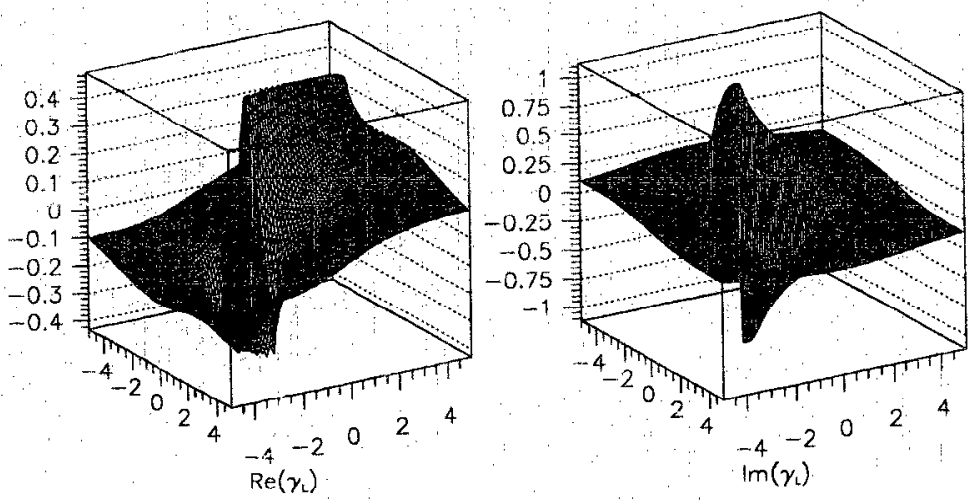

Fig. 1. The BFKL anomalous dimension $\gamma_{\mathrm{L}}$ as a function of the complex moment variable $\omega / \bar{\alpha}_{S}$ : (a) Real part, (b) Imaginary part.

$\gamma_{\mathrm{L}}=\left(\begin{array}{cc}0 & 0 \\ \frac{C_{F}}{C_{A}} \gamma_{\mathrm{L}}(\omega) & \gamma_{\mathrm{L}}(\omega)\end{array}\right)+\mathcal{O}\left(\alpha_{S}\left(\alpha_{S} / \omega\right)^{k}\right)$,

where the BFKL anomalous dimension $\gamma_{\mathrm{L}}(\omega)$ is determined by the implicit equation

$\omega=\bar{\alpha}_{S} \chi\left(\gamma_{\mathrm{L}}\right), \bar{\alpha}_{S} \equiv C_{A} \alpha_{S} / \pi$
$\chi(\gamma) \equiv 2 \psi(1)-\psi(\gamma)-\psi(1-\gamma)$,

$\psi$ being the Euler $\psi$-function. At large values of $\omega / \bar{\alpha}_{S}$, the solution of Eq. (8) $\mathrm{crn}$ be obtained by expanding the characteristic function $\chi(\gamma)$ for small values of $\gamma$. The first terms of the power series expanision in $\alpha_{S}$ for $\gamma_{\mathrm{L}}(\omega)$ are

$\gamma_{L}(\omega)=\frac{\bar{\alpha}_{S}}{\omega}+2.40\left(\frac{\bar{\alpha}_{S}}{\omega}\right)^{4}+\mathcal{O}\left(\frac{\bar{\alpha}_{S}}{\omega}\right)^{5}$.

In the region of sniall $x$, however, smaller and smaller values of $\omega / \bar{\alpha}_{S}$ are probed, and a full inversion of Eq. (8) has to be performed. This procedure leads to a many-valued function of the complex moment variable $\omega$. The perturbative branch of $\gamma_{L}(\omega)$ is defined by requiring it to match the expansion (9) at large $\omega$. The full solution, found numerically using Newton's method, is shown in Fig. 1. We see that the all-order resummation of the perturbative $\omega$-poles builds up a branch-point singularity at the value $\omega_{\mathrm{L}}=4 \ln 2 \bar{\alpha}_{S} \simeq$ $2.77 \bar{\alpha}_{S}$ in the complex $\omega$-plane. Correspondingly, as $\omega$ decreases through $\omega_{\mathrm{L}}$ the BFKL anomalous dimension $\gamma_{L}(\omega)$ departs from the fixed-order behaviour: its real part rises quickly until it saturates at the value
$\gamma_{\mathrm{L}}=\frac{1}{2}$, and its imaginary part develops a discontinuity along the real axis. In $x$-space, this singularity of the anomalous dimension gives rise $t$ a power-like behaviour $x^{-\omega_{1}}$ of the gluon density at asymptotically small values of $x$ [3]. The only other singularities of $\gamma_{\mathrm{L}}(\omega)$ on the perturbative sheet are a complex conjugate pair of branch-points at $\omega=(-1.41 \pm 1.97 i) \bar{\alpha}_{S}$ [17]. We avoid these singularities when choosing a contour for the inversion of Eq. (2).

At the next-to-leading logarithmic $(\mathrm{NL}(x))$ level, quarks start to contribute to the evolution at small $x$ on the same footing as gluons. The $\mathrm{NL}(x)$ contribution to the singlet anomalous dimension matrix can be written as

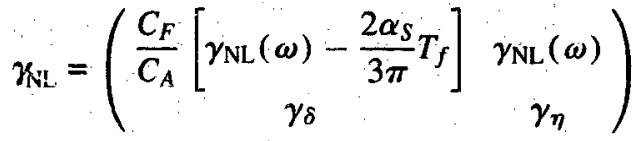

$$
\begin{aligned}
& +\mathcal{O}\left(\alpha_{S}^{2}\left(\alpha_{S} / \omega\right)^{k}\right)
\end{aligned}
$$

$\left(T_{f}=T_{R} N_{f}=\frac{1}{2} N_{f}\right)$, where the corrections $\gamma_{\delta}, \gamma_{\eta}$ to the gluon anomalous dimensions are unknown at present, while the contributions $\gamma_{\mathrm{NL}}(\omega)$ to the quark anomalous dimensions have recently been computed $[12,13]$. In the DIS factorization scheme [18] $\gamma_{\mathrm{NL}}(\omega)$ is given explicitly in terms of the BFKL anomalous dimension $\gamma_{\mathrm{L}}(\omega)$ as ${ }^{2}$

\footnotetext{
${ }^{2}$ Formuiae are also given in Refs. $[12,13]$ for the $\overline{\mathrm{MS}}$ scheme [19]. Results for both schemes will be presented in Ref. [17].
} 


$$
\begin{aligned}
& \gamma_{\mathrm{NL}}^{\mathrm{DIS}}\left(\omega, \alpha_{S}\right)=\frac{\alpha_{S}}{\pi} T_{f} \frac{2+3 \gamma_{\mathrm{L}}-3 \gamma_{\mathrm{L}}^{2}}{3-2 \gamma_{\mathrm{L}}} \\
& \therefore \times \frac{\Gamma^{3}\left(1-\gamma_{\mathrm{L}}\right) \Gamma^{3}\left(1+\gamma_{\mathrm{L}}\right)}{\Gamma\left(2+2 \gamma_{\mathrm{L}}\right) \Gamma\left(2-2 \gamma_{\mathrm{L}}\right)} R\left(\gamma_{\mathrm{L}}\right)
\end{aligned}
$$

where

$$
\begin{aligned}
R(\gamma) \equiv\left\{\frac{\Gamma(1-\gamma) \chi(\gamma)}{\Gamma(1+\gamma)\left[-\gamma \chi^{\prime}(\gamma)\right]}\right\}^{\frac{1}{2}} \\
\quad \times \exp \left\{\gamma \psi(1)+\int_{0}^{\gamma} d \zeta \frac{\psi^{\prime}(1)-\psi^{\prime}(1-\zeta)}{\chi(\zeta)}\right\}
\end{aligned}
$$

$\Gamma$ being the Euler $\Gamma$-function, and $\chi, \chi^{\prime}$ th characteristic function in $\mathrm{Eq} .(8)$ and its first derivative, respectively. Eq. (11) introduces no singularities in the $\omega$-plane to the right of $\omega_{\mathrm{L}}$. As in the BFKL case, at moderate values of $\omega,|\omega|>\omega_{\mathrm{L}}$, an accurate representation of the quark anomalous dimension can be obtained by taking the first few perturbative contributions to Eq. (11),

$$
\begin{aligned}
\gamma_{\mathrm{NL}}^{\mathrm{DIS}} & =\frac{2 \alpha_{S}}{3 \pi} T_{f}\left\{1+2.17 \frac{\bar{\alpha}_{S}}{\omega}+2.30\left(\frac{\bar{\alpha}_{S}}{\omega}\right)^{2}\right. \\
& \left.+8.27\left(\frac{\bar{\alpha}_{S}}{\omega}\right)^{3}+\mathcal{O}\left(\frac{\bar{\alpha}_{S}}{\omega}\right)^{4}\right\}
\end{aligned}
$$

Note that, unlike the expansion (9) for $\gamma_{L}$, all the perturbative coefficients in Eq. (12) are non-vanishing, suggesting an earlier departure from the lowest-order behaviour in the quark sector $[12,4]$. At very small values of $x$, the fully resummed result (11) has to be used. As $\gamma_{L}(\omega)$ approaches its saturation value at $\gamma=\frac{1}{2}$, the quark anomalous dimension (11) increases rapidly, with the behaviour $1 /\left(1-2 \gamma_{L}\right)^{1 / 2}$, leading to stronger scaling violation.

\section{Parton evolution}

We now consider the evolution as determined by the resummed kernels described in Section 2. We match the BFKL anomalous dimensions (7) and the nextto-leading-logarithmic results (10) with the full one[15] and two-loop [16] expressions, subtracting the resummed terms to avoid double counting, as follows

$$
\begin{gathered}
\gamma_{a b}^{\mathrm{NLO}}\left(\omega, \alpha_{S}\right)=\gamma_{a b}^{(1)}(\omega) \alpha_{S}+\gamma_{a b}^{(2)}(\omega) \alpha_{S}^{2} \\
+\sum_{k=3}^{\infty}\left(\frac{\alpha_{S}}{\omega}\right)^{k} A_{a b}^{(k)}+\sum_{k=2}^{\infty} \alpha_{S}\left(\frac{\alpha_{S}}{\omega}\right)^{k} B_{a b}^{(k)}
\end{gathered}
$$

We evaluate the running coupling at two-loop level from Eq. (3). Since resummed expressions for the next-to-leading gluonic contributions $\gamma_{\delta}, \gamma_{\eta}$ in Eq. (10) are not yet available, we set them equal to their two-loop values. We call the resulting expressions the next-to-lead'ng quark $(\operatorname{NLQ}(x))$ approximation. For deep inelastic lepton scattering, the quark nomalous dimensions are more relevant, because the stucture functions couple directly to quarks, whereas they couple to gluons via an $\mathcal{O}\left(\alpha_{S}\right)$-suppresse ${ }^{1}$ coefficient function:

One should note that small- $x$ resummation does not preserve the momentum sum rules $\sum_{a} \gamma_{a b}(\omega=$ $1, \alpha_{S} j=0$, because it neglects all terms other than those singular at $\omega=0$. Momentum conservation can be restored to all-loop order by introducing contributions to the anomalous dimensions which are subdominant at $\omega \rightarrow 0$ (leaving the leading and next-toleading logarithmic series unchanged) but enforce the correct behaviour at $\omega \rightarrow 1$. We do this by multiplying the resummed anomalous dimensions by an overall factor of $(1-\omega)$. To test the sensitivity of the results to this prescription, we shall also show results using a 'harder' model of momentum conservation, in which we simply subtract from each resummed expression a constant equal to its value at $\omega=1$.

We have solved the evolution equations assuming various alternative sets of parton distributions as input conditions at scale $Q_{0}^{2}=4 \mathrm{GeV}^{2}$. In this Letter we limit ourselves to the case of the flat MRSDO' [20] input distributions. Flat parton distributions at low $Q^{2}$ are suggested by the fixed-target data of the E665 Collaboration [21], and it is of particular interest to see whether they could be consistent with the steep rise at small $x$ seen at higher $Q^{2}$ at HERA [22]. Data at low $Q^{2}$ and small $x$ from HERA should also be available soon. We refer to Ref. [17] for a more complete discussion including different inputs.

The resulting DIS-scheme singlet densities are shown in Fig. 2 as functions of $x$ for different values of the hard scale $Q^{2}$ Comparison with the fixed-order prediction shows that the effects of the $L(x)$ BFKL resummation set in at $x$-values of the order of $10^{-3}$. 


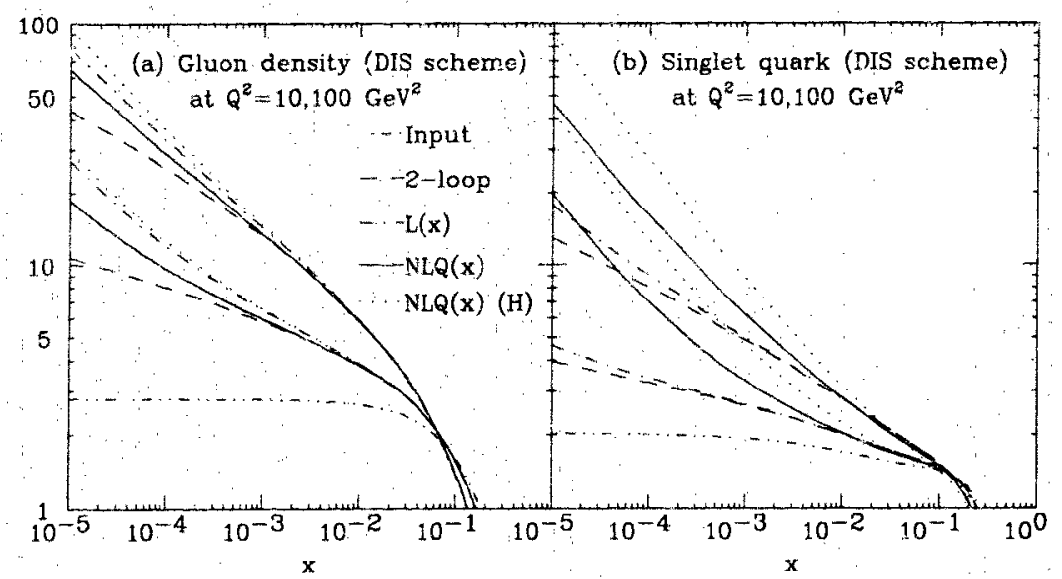

Fig. 2. (a) Gluon and (b) quark singlet density in the DIS-scheme at different values of $Q^{2}\left(Q^{2}=10,100 \mathrm{GeV}^{2}\right)$ as ottained from two-loop (dashed), two-loop $+\mathrm{L}(x)$ (doi-dashed), two-loop $+\mathrm{NLQ}(x)$ (solid) evolution of flat input distributions $\left(\mathrm{MRSDO}^{\prime}\right)$ at $Q_{0}^{2}=4$ $\mathrm{GeV}^{2}$. The dotted curves correspond to $\mathrm{NLQ}(x)$ resummation with the harder model for momentum conservation described in the text.

They increase as $x$ becomes smaller, and are still moderate in the range $\left(x \lesssim 10^{-4}\right)$ accessibie at present colliders. This is in qualitative agreement with earlier estimates based on models which seek to extend the BFKL equation to include a running coupling ' 8,23 ].

We see, on the other hand, that in the range of $x$ and $Q^{2}$ values shown, the resummation effects from quark evolution are quite sizeable, and tend to $h$ larger than those in the gluon channel, in spite of their being formally sub-leading. In particular, they set in quite early in $x$, around values of $x \sim 10^{-2}$. This is in agreement with expectations based on comparing the perturbative expansions (9) and (12).

It should be noted, however, that the impact of momentum-conserving corrections is modeldependent. The difference between the results obtained using our preferred procedure and the harder model provides an indication of the theoretical uncertainty on the present predictions at small $x$ due to unknown sub-dominant contributions.

The results described above can be used directly to obtain resummed predictions for the deep inelastic structure function $F_{2}$. The $\mathrm{NLQ}(x)$ result; again for the case of the flat MRSD0 input distributions, is the solid curve reported in Fig. 3. The 1993 ZEUS data [24] are also s lown for reference purposes. We see that a steep rise of $F_{2}$ at small $x$ is already obtained in the HERA range, starting from flat input distributions, as a consequence of perturbative resummation. As in
Fig. 2, the next-to-leading corrections in the quark sector have a far more dramatic impact than the BFKL contributions.

\section{Summary}

We have performed a study of QCD dynamics at small $x$ by incorporating resummed anomalous dimensions into the renormalization group evolution equations. We have used the resummed equations to determine predictions for the singlet parton densities and for the deep inelastic structure function $F_{2}$.

Our results suggest that the fixed-order evolution of parton densities which are flat at small $x$ for $Q_{0}^{2} \sim 4$ $\mathrm{GeV}^{2}$ is a poor approximation. Effects from leading and next-to-leading resummation at small $x$ are already sizeable in the HERA region, and give rise to a steep increase of the structure function $F_{2}$. In particular, the dominant contribution to this behaviour comes from the next-to-lcading corrections in the quark channel.

At the moment, the resummed predictions are still subject to large uncertainties, from two sources. First, the next-to-leading calculation is not complete: the gluonic contributions beyond two-loop order are unknown. Second, subdominant contributions, such as those responsible for momentum conservation beyond two-loop order, are still important at HERA energies. 


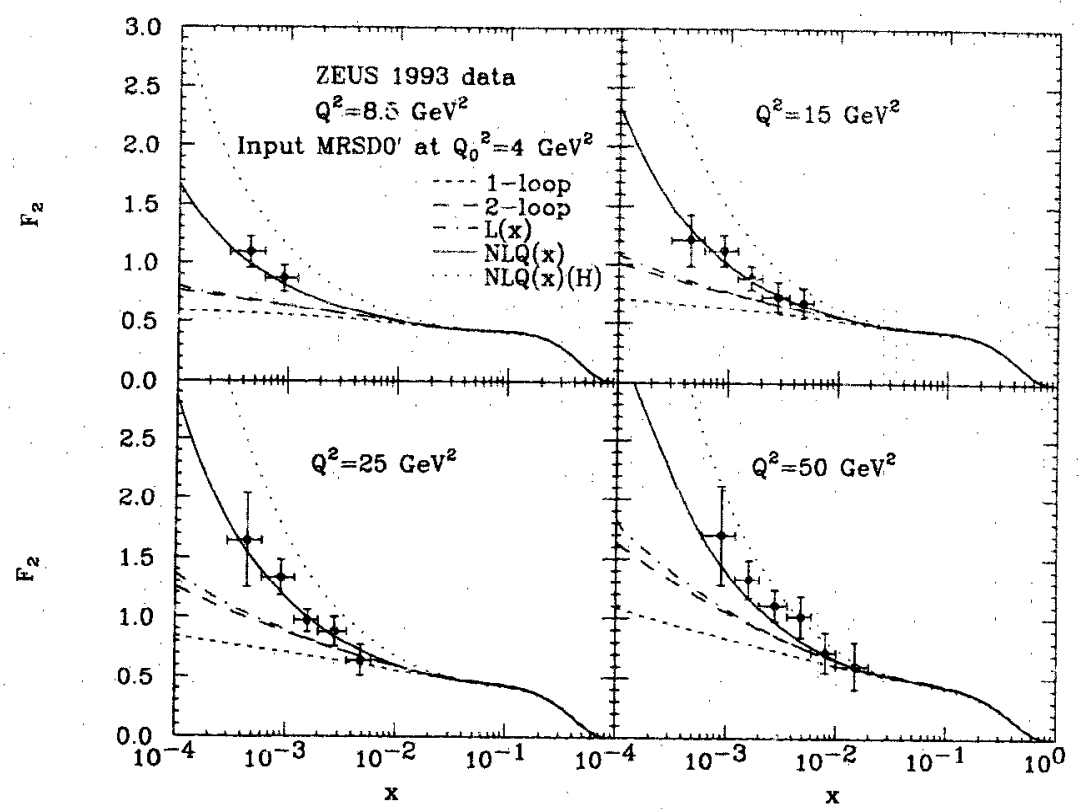

Fig. 3. Resummed predictions for the structure function $F_{2}$. The short-dashed curve is the one-loop fixed-order prediction. The other curyes are as in Fig. 2.

\section{Acknowledgments}

We are most grateful to Stefano Catani for a number of valuable discussions on topics related to this work. We would like to thank Gavin Salam for help in preparing Fig. 1. R.K.E. would like to hank the Cavendish and Rutherford-Appicion Laboratories for their hospitality, and the UK Particle Physics and Astronomy Research Council for the award of a Senior Fellowship. F.H. acknowledges the hospitality of the Theory Group at Fermilab.

\section{References}

[1] See, for instance, W.J. Stirling, in Proceedings of the Aachen Conference: QCD - 20 years later, eds. P.M. Zerwas and H.A. Kastrup (World Scientific, Singapore, 1993) p. 387.

121 J. Feltesse, Saclay preprint Dapnia/SPP 94-35, rapporteur talk at the 27th International Conference on High Energy Physics, Glasgow, July 1994 .

[3] For recent reviews see, for instance, J. Kwiecinski, Krakow Report No. 1681/PH, to appear in Pro sedings of the Workshop QCD94, Montpcllier, July 1994;

S. Catani, Florence preprint DFF $207 / 6 / 94$, to appear in Proceedings of Les Rencontres de Physique de la Vallee d'Aoste, La Thuile, March 1994.
[4] R.K. Ellis, Z. Kunszt and E.M. Levin, Nucl. Phys: B 420 (1994) 517.

[5] L.N. Lipatov, Sov, J. Nucl. Phys. 23 (1976) 338;

E.A. Kuraev, L.N. Lipatov and V.S. Fadin, Sov. Phys. JETP 45 (1977) 199;

Ya. Balitskii and LN. Lipatov, Sov. J. Nucl. Phys. 28 (1978) 822.

[6] V.S. Fadin and L.N. Lipatov, Nucl. Phys. B 406 (1993) 259.

[7] A.R. White, Phys Lett. B 334 (1994) 87.

[8]' J. Kwiecinski, Zeit. Phys. C 29 (1985) 561.

[9] L.V. Gribov, E.M. Lcvin and M.G. Ryskin, Phys. Rep. 100 (1983) 1;

E.M. Levin and M.G. Ryskin, Phys. Rep. 189 (1990) 268.

[10] A.J. Askew; J. Kwiecinski, A.D. Martin and P.J. Sutton, Phys. Rev. D 47 (1993) 3775; 49 (1994) 4402.

[11] S. Catani, M. Ciafaloni and F. Hautmann, Phys. Lett. B 242 (1990) 97; 307 (1993) 147; Nucl. Phys. B 366 (1991) 135

[12] S. Catani and F. Hautmann, Phys. Lett. B 315 (1993) 157.

[13] S. Catani and F. Hautmann, Nucl. Phys. B 427 (1994) 475 .

[14] J. Bartels, Phys. Lett. B 298 (1993) 204; Zeit. Phys. C 60 (1993) 471;

A.H. Mueller, Nucl. Phys. B 335 (1990) 115, preprint CUTP-640.

[15] V.N. Gribov and L.N Lipatov, Sov. J Nicl. Phys 15 (1972) 438, 675;

G. Altarelli and G. Parisi, Nucl Phys. B 126 (1977) 298; Yu.L. Dokshitzer, Sov. Phys. JETP 46 (1977) 641.

[16] E.G. Floratos, D A Ross and C.T. Sachrajda, Nucl Phys. B 1.2 (1979) 493;

G. Curci, W: Furmanski and R. Petronzio, Nucl. Phys. B 
175 (1980) 27 ;

W. Furmanski and R. Petronzio, Phys. Lett. B 97 (1980) 437:

E.G. Floratos, C. Kotnnas and R. Lacaze, Nucl. Phys. B $192(1981) 417$.

[17] R.K. Ellis, F. Hautmann and B.R. Webher, Cambridge preprint Cavendish-HEP-94/20, in preparation.

[18] G. Altarelli, R.K. Ellis and G. Martinelli, Nucl. Phys. B 157 (1979) 461 .

[19] W.A. Bardeen, A.J Buras, D.W. Duke and T. Muta, Phys. Rev. D 18 (1978) 3998.

[20] A.D. Martin, R.G. Roberts and W.J. Stirling, Phys. Lett. B $306(1993) 145$
[21] A.V. Kotval, preprint Fermilat-Conf-94/345-E, to be published in Proc. VIth Rencontres de Blois, Blois, June 1994.

[22] ZEUS Collaboration; M. Derrick et al,, Phys. Lett. B 316 (1993) 412;

H1 Collaboration, I. Abt et al., Nucl. Phys. B 407 (1993) 515; Phys. Lett. B 321 (1994) 161.

[23] E.M. Levin, G. Marchesini, M.G. Ryskin and B.R. Webber, Nucl: Phys. B 357 (1991) 167.

[24] ZEUS Collaboration, preprint DESY-94-143. 